Oral presentation

\title{
Trauma systems and early management of severe injuries in Scandinavia: review and current status
} Thomas Kristiansen*1,5, Kjetil Søreide ${ }^{2,3}$, Kjetil Ringdal1,5, Marius Rehn1,5, Andreas J Krüger ${ }^{1}$, Andreas Reite ${ }^{2}$, Terje Meling ${ }^{4}$, Pål Aksel Næss ${ }^{6}$ and Hans Morten Lossius ${ }^{1}$

\author{
Address: ${ }^{1}$ Norwegian Air Ambulance Foundation, Department of Research, Drøbak, Norway, ${ }^{2}$ Department of Surgery, Stavanger University \\ Hospital, Norway, ${ }^{3}$ Department of Surgical Sciences, University of Bergen, Norway, ${ }^{4}$ Department of Orthopaedic Surgery, Stavanger University \\ Hospital, Norway, ${ }^{5}$ Faculty of Medicine, Faculty Division Ullevål University Hospital, University of Oslo, Norway and ${ }^{6}$ Trauma Unit, Oslo \\ University Hospital Ullevål, Oslo, Norway \\ Email: Thomas Kristiansen* - thomas.kristiansen@snla.no \\ * Corresponding author
}

from Scandinavian Update on Trauma, Resuscitation and Emergency Medicine 2009

Stavanger, Norway. $23-25$ April 2009

Published: 28 August 2009

Scandinavian Journal of Trauma, Resuscitation and Emergency Medicine 2009, I7(Suppl 3):O29 doi:I0.I I86/I757-724I-17-S3-O29

This abstract is available from: http://www.sjtrem.com/content/I7/S3/O29

(C) 2009 Kristiansen et al; licensee BioMed Central Ltd.

\section{Introduction}

Scandinavian countries face common challenges in trauma care. There are suggestions that Scandinavian trauma system development is immature compared to other regions. We wanted to assess the current status of Scandinavian trauma management and system development.

\section{Methods}

An extensive search of the Medline/Pubmed, EMBASE and SweMed+ databases was conducted. A wide coverage was prioritized over systematic search strategies. Scandinavian publications pertaining to trauma epidemiology, trauma systems and early trauma management from the last decade were included.

\section{Results}

The incidence of severe injury range from 30 to 52 per 100,000 per yr, with about $90 \%$ due to blunt trauma. Parts of Scandinavia are sparsely populated and with long prehospital distances. In accordance with other European countries, prehospital physicians are widely employed and studies indicate consequent survival benefits in trauma patients. More than 200 Scandinavian hospitals receive injured patients, increasingly with multidiscipli- nary trauma teams. Challenges remain in the prehospital identification of the severely injured. Improved triage allows for better match between patient needs and level of resources made available. Trauma management is threatened by the increasing sub-specialization of professions and institutions. Scandinavian research is leading the development of team- and stimulation-based trauma training. Several pan-Scandinavian co-efforts have facilitated research and provided guidelines for clinical management.

\section{Conclusion}

Scandinavian trauma research is characterized by an active collaboration across countries. The challenges met require an increased focus on the role of traumatology within an increasingly fragmented health care system. Regional networks of predictable and accountable pre and in-hospital resources are needed for efficient trauma systems. Successful developments require novel research and scientific assessment of imported principles. 